\title{
The distance to NGC 5904 (M 5) via the subdwarf main sequence fitting method ${ }^{\star}$
}

\author{
V. Testa ${ }^{1}$, A. Chieffi ${ }^{2}$, M. Limongi ${ }^{1}$, G. Andreuzzi ${ }^{1,3}$, and G. Marconi ${ }^{1,4}$ \\ 1 INAF - OAR, via Frascati 33, 00040 Monteporzio Catone, Italy \\ 2 IASF - CNR, via del Fosso del Cavaliere, 00100, Roma, Italy \\ 3 INAF - TNG, Santa Cruz de La Palma, Canary Islands, Spain \\ ${ }^{4}$ ESO - Chile, Alonso de Cordova 3107, Vitacura, Casilla 19001, Santiago 19, Chile
}

Received 13 October 2003 / Accepted 4 March 2004

\begin{abstract}
We present a determination of the distance modulus of the globular cluster NGC 5904 (M 5), obtained by means of the subdwarf main-sequence fitting on the $(V, V-I)$ color-magnitude diagram. The subdwarf sample has been selected from the HIPPARCOS catalog in a metallicity range homogeneous with the cluster $([\mathrm{Fe} / \mathrm{H}] \simeq-1.1)$. Both the cluster and the subdwarfs have been observed with the same telescope+instrument+filters setup (namely, ESO-NTT equipped with the SUSI2 camera), in order to preserve homogeneity and reduce systematic uncertainties. A set of archival HST data has then been used to obtain a deep and precise ridge line. These have been accurately calibrated in the ground photometric system by using the NTT data and used to fit the cluster distance modulus. By adopting the most commonly accepted values for the reddening, $E(B-V)=0.035$ and 0.03 , we obtain respectively $\mu_{0}=14.44 \pm 0.09 \pm 0.07$ and $\mu_{0}=14.41 \pm 0.09 \pm 0.07$, in agreement with recent determinations.
\end{abstract}

Key words. Galaxy: globular clusters: individual: NGC 5904 (M 5) - stars: distances

\section{Introduction}

The ages of the Galactic Globular Clusters (hereafter GGCs) represent one of the milestones for our comprehension of the Universe. To derive reliable absolute ages estimates, the most important parameter to determine is the distance (see Renzini 1991). Errors on the distance propagate through the age determination procedure such that a 0.08 error in the distance modulus (DM) gives a $12 \%$ error in the age. Different ages (younger or older) mean different epochs of formation of the Milky Way. Therefore, it can be easily seen how the uncertainty in GGC age estimates significantly affects models of galaxy formation and cosmological evolution.

The recent availability of the HIPPARCOS catalog of trigonometric parallaxes (Perryman et al. 1997) has greatly improved knowledge of GGC distances. A large number of papers on the subject have been published since then (e.g., Reid 1997; Gratton et al. 1997; Pont et al. 1998; Vandenberg et al. 2002; Gratton et al. 2003), most of which present larger distances, and hence younger ages, with respect to previous work (with the exception, among the cited works, of Pont et al. 1998, who recover the pre-Hipparcos results of 1992).

Send offprint requests to: $\mathrm{V}$. Testa,

e-mail: testa@mporzio.astro.it

* Based on data collected at ESO-La Silla, Chile, (GTO 63.L-0717) and from HST archival data (GO 8310).
The method used consists of a fit of the cluster main sequence to the subdwarf main-sequence of corresponding metallicity, once the reddening has been subtracted. This procedure is, of course, prone to uncertainties, both random and systematic: on the subdwarf side errors may come from parallax measurements, Lutz-Kelker correction and metallicity; on the cluster side reddening, metallicity, narrowness and accuracy of the mean ridge line influence the results. A further effect is represented by the presence of binary stars in the cluster CMD and in the subdwarf sample. In the first case the determination of the mean ridge line is altered, if a proper fitting procedure is not taken into account. In the second, the magnitude of the field star is different from stars of analogous metallicity. This issue has been widely discussed in, e.g., Gratton et al. (1997); Pont et al. (1998); Carretta et al. (2000), and will be analyzed in detail later. If HIPPARCOS allowed us to significantly reduce the random errors on parallax measurements, nonetheless other sources of error still remain. Metallicity, for example, represents a common problem that significantly affects the determination of the distance modulus, because of either the spread in metallicity of the sequence of local subdwarfs or the error on the absolute values. There are two strategies to avoid this problem: to obtain a sufficiently large set of subdwarfs in the right metallicity range, or to apply a relation that transforms the colors of the stars of different metallicity. The second introduces a model-dependent relation and should be used with caution while the first is better, especially if the subdwarf 
sample extends over the main sequence down to very low masses. In fact, recent HST color-magnitude diagrams of GGCs reach very faint magnitudes, hence small masses, but a similar sequence for subdwarfs is currently not available. Reid \& Gizis (1998) fit a distance modulus to NGC 6397 by using a sequence of lower MS subdwarfs, but their work is based on the fact that the sequence of very metal-poor subdwarfs define a reasonably narrow sequence. This is not true, instead, for subdwarfs of higher metallicities (see their Fig. 2). On the other hand, the most widely used set of subdwarfs cover magnitudes ranging from $M_{V} \sim 7$ and brighter, leaving uncovered the range between this value and the extreme subdwarfs. This is explained by the relative lack of halo stars near the Sun (Reid $\&$ Gizis 1998). One of the possibilities we discuss to reduce the uncertainties is to perform a uniform study of a subdwarf sample and a GGC using the same telescope, instrumentation and, possibly, observing nights, thus eliminating the systematics due to the relative calibrations among different instrumental setups. For this reason, we observed the target cluster and a sample of subdwarfs in the proper metallicity range matching the cluster's with the same telescope+instrument (NTT+SUSI2) and then used archival data from HST for faint stars, and calibrated the HST magnitudes to the ground system, obtaining a deep and fully homogeneous data set. The selected object is the well-known cluster NGC 5904 (M 5), for which a wide set of photometric and spectroscopic studies is available in the literature. Moreover, Sandquist et al. (1996, S96), Reid (1997), Gratton et al. (1997), Chaboyer et al. (1998) and more recently, Vandenberg et al. (2002) determined the DM of the cluster by using the trigonometric parallaxes of subdwarfs and various sets of models. These works will be often referred to in the following.

NGC 5904 is a typical old halo cluster of intermediate metallicity $-[\mathrm{Fe} / \mathrm{H}] \sim-1.1$ in the scale of Carretta \& Gratton (1997) (CG), -1.4 in the Zinn \& West (1984) scale (ZW). A recent study of Ramirez \& Cohen (2003) gives $[\mathrm{Fe} / \mathrm{H}] \simeq-1.3$. The morphology of the horizontal branch (HB) does not show signs of the second parameter effect and several age determinations are available. The structure of the paper is as follows: Sect. 2 describes the observations and the archival material, Sect. 3 presents the reduction and calibration of the data, in Sect. 4 we present and analyze the subdwarf sample. In Sect. 5 the cluster color-magnitude diagram (CMD) is presented and discussed, and in Sect. 6 the DM-fit is analyzed. In Sect. 7 a final discussion is summarized.

\section{Data set}

\subsection{Observations}

Observations have been performed with the ESO-NTT telescope equipped with SUSI2 as a backup program during three different campaigns, in December 1999 (63.L-0717, subdwarfs + cluster), December 2000 (66.D-0242, subdwarfs), February 2002 (68.B-0061, cluster). The adopted strategy was to observe both the subdwarf sample and the target cluster with the same telescope+instrument+filters+detector setup and, possibly, within the same campaign, to remove any systematic effect due to the use of different setups. The December 1999 and February 2002 campaigns produced very good quality data, while the December 2000 set (two subdwarfs only) presented moderately poor photometric conditions. Seeing was not a major problem in observing the subdwarfs, because they had to be defocused so as not to saturate the chip. This, coupled to the fact that the objects were observed in the central region of the frames, allowed us to use exposure times long enough to have a negligible shutter effect. The typical observing time per frame was $0.5 \mathrm{~s}$.

The data set for the target cluster $($ NGC $5904=$ M 5) was secured in December 1999, when the seeing was poor, together with the subdwarfs, and in February 2002 when seeing was considerably better, to have a comparison data-set. In both cases, the run was found to be photometric and the runto-run scatter in the magnitudes is almost absent, without any trend in the magnitudes and colors (see Fig. 5). The field was selected 2 arcmin North of the cluster center, to ensure an almost complete overlap with the fields observed with HST that were retrieved from the archive (see below). Only images in $V$ and $I$ bands for the cluster were taken, because the spacebased data-set consists of images in these two bands only. For the subdwarfs, B frames also were secured.

\subsection{Archival data}

A set of deep exposures was available from the STScI HST WFPC2 archive (GO 8310) in the two wide bands $F 555 W(V)$ and $F 814 W(I)$. A total of $6 F 555 W$ and $9 F 814 W$ frames were retrieved from the archive, and pre-reduced using the HST pipeline, available in STSDAS running under IRAF (Tody $1986,1993)^{1}$, and using the most recent calibration frames and tables (see the WFPC2 web page for details).

\section{Reductions and calibrations}

\subsection{Reductions}

Reductions were done for all the data-sets by using IRAF and the digiphot packages daophot and photcal for building the catalog and apply photometric calibrations respectively. In particular, since the telescope had been defocused when observing the subdwarf sample, for these stars aperture photometry has been used. For cluster images, both NTT and HST, we applied the standard daophot PSF fitting routines. In particular, the NTT data-set is characterized by a relatively high oversampling ( $F W H M \sim 5$ pix), and in this case a Gaussian model for the PSF plus look-up table of correction varying quadratically along the frames has been used. HST-WFPC2 frames, on the other hand, are undersampled (estimated $F W H M \sim 1.6$ pix), and in this case a moffat 15 function, with quadratic look-up table, gave better results. As a general strategy, object searching was done independently on each frame, after registering all of them to a common reference frame (the best seeing one).

\footnotetext{
${ }^{1}$ IRAF is distributed by the National Optical Astronomy Observatories, which are operated by the Association of Universities for Research in Astronomy, Inc., under cooperative agreement with the National Science Foundation.
} 
Table 1. Calibration coefficients for the three runs.

\begin{tabular}{clcc}
\hline \hline Filter & Zero point & Color term & Extinction \\
\hline \multicolumn{5}{c}{ Dec. 1999 } & \\
$B$ & $25.42(0.01)$ & $-0.05(0.01)$ & $0.23(0.01)$ \\
$V$ & $25.82(0.01)$ & $0.02(0.01)$ & $0.11(0.01)$ \\
$I$ & $24.74(0.01)$ & $-0.04(0.01)$ & $-0.04(0.01)$ \\
\multicolumn{5}{c}{ Dec. 2000 } & \\
$B$ & $25.86(0.03)$ & $-0.18(0.04)$ & $0.29(0.02)$ \\
$V$ & $25.94(0.04)$ & $0.00(0.04)$ & $0.19(0.03)$ \\
$I$ & $24.80(0.01)$ & $-0.17(0.01)$ & $-0.09(0.02)$ \\
\multicolumn{5}{c}{ Feb. 2002 } & & \\
$V$ & $25.69(0.01)$ & $-0.01(0.01)$ & $0.15(0.01)$ \\
$I$ & $24.59(0.02)$ & $-0.03(0.01)$ & $-0.06(0.01)$ \\
\hline
\end{tabular}

In the case of HST, all the images were almost perfectly registered onto each other, and no shift or rotation was needed. We made use of the world coordinate system available in the FITS header in order to obtain later an astrometric calibration of the catalogs.

\subsection{Photometric calibrations}

For both data-sets, aperture corrections were applied to convert the PSF magnitudes to aperture magnitudes before calibrating. HST-WFPC2 magnitudes were then normalised to the standard aperture of $0.1^{\prime \prime}$, following the calibration procedure of Holtzmann et al. (1995). For ESO-NTT data, growthcurves (from DAOGROW) were used and aperture corrections at infinity computed. For ground-based data, a set of Landolt standards was secured for each run and calibration equations, having form (1), derived. Table 1 reports the calibration coefficients of all the runs. The equation for the $V$ filter was derived against $B-V$ in the two first runs, as $B, V, I$ data were available both for subdwarfs and for NGC 5904. In the last run, only NGC 5904 was observed and only in $V$ and $I$ filters, hence we calibrated the $V$ magnitude against $(V-I)$.

$$
\begin{aligned}
v & =V+a_{0}+a_{1} \times(b-v)+a_{2} \times X_{V} \\
v & =V+a_{0}^{\prime}+a_{1}^{\prime} \times(v-i)+a_{2}^{\prime} \times X_{V} \\
b & =B+b_{0}+b_{1} \times(b-v)+b_{2} \times X_{B} \\
i & =I+c_{0}+c_{1} \times(v-i)+c_{2} \times X_{I} .
\end{aligned}
$$

Figures 1-3 show the difference between standard mags. and calibrated mags. of the Landolt stars used for calibration in all the runs. As can be seen, the residuals $\left(\mathrm{Mag}_{\text {std }}-\mathrm{Mag}_{\mathrm{fit}}\right)$ are on average very small and with no residual trends with the colors.

The HST-WFPC2 data-set was calibrated following the procedure describred in Holtzmann et al. (1995). After applying the aperture corrections and correcting for geometric distorsions and CTE effect, following Dolphin (2000), we applied the published coefficients and calibrated the data-set both in the VEGAMAG and STMAG systems. While the $V$ magnitudes are almost identical in the two systems, the $I$ magnitudes differ by a certain amount (around $1.2 \mathrm{mag}$, depending on the WF chip).
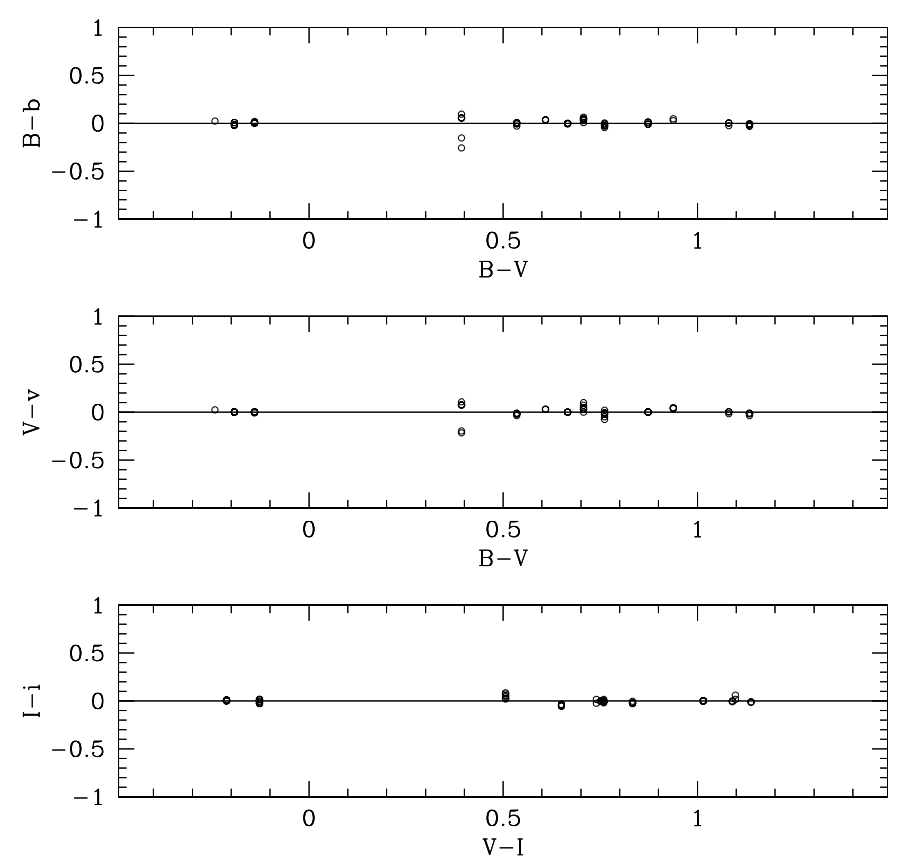

Fig. 1. Residual between standard magnitudes and calibrated magnitudes for the Landolt stars in the Dec. 1999 run.
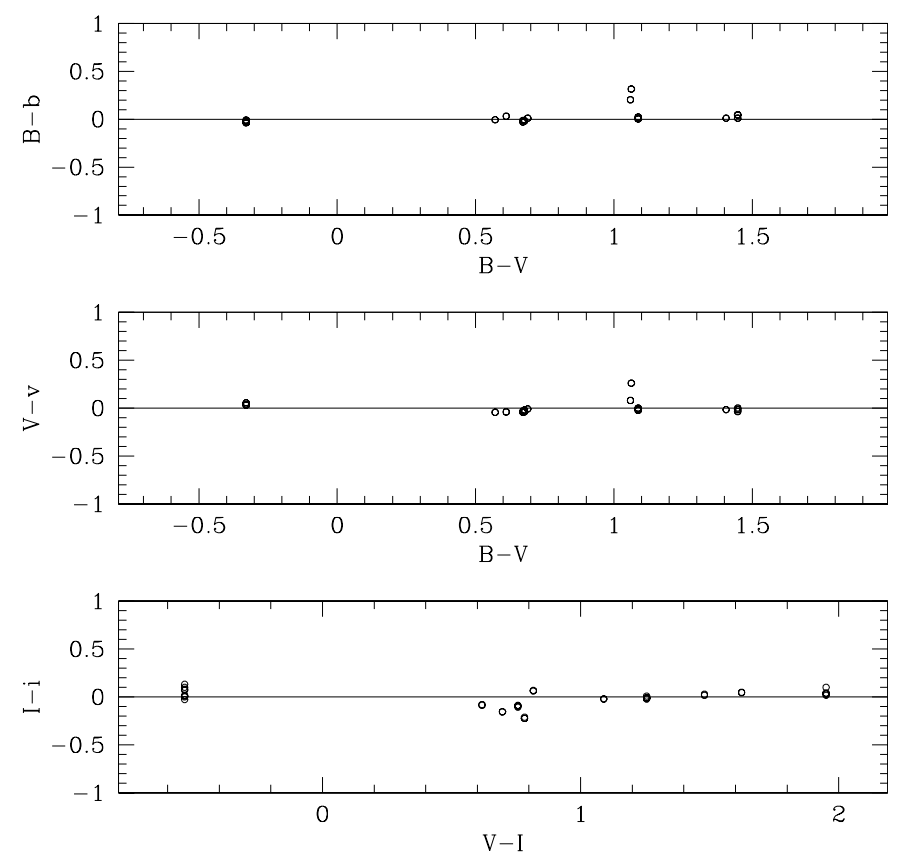

Fig. 2. Residual between standard magnitudes and calibrated magnitudes for the Landolt stars in the Dec. 2000 run.

The NTT data-set was then used to provide magnitudes in both system to the ground NTT system, as an internal check.

\section{The subdwarf sample}

The sample of subdwarfs used for this study was selected from the HIPPARCOS list taken from the papers of Gratton et al. (1997) and Reid (1997). The requirement was that the sample be as close as possible in metallicity to the target cluster, in order to reduce systematics introduced by any transformation that 
Table 2. The subdwarf sample.

\begin{tabular}{lrrrrrrrrrc}
\hline \hline Ident. & $B$ & $\sigma(B)$ & $V$ & $\sigma(V)$ & $I$ & $\sigma(I)$ & {$[\mathrm{Fe} / \mathrm{H}]$} & $M_{v}$ & $\pi_{\text {mas }}$ & Run \\
\hline HIP70681 & 9.881 & 0.001 & 9.302 & 0.001 & 8.577 & 0.001 & $-1.09 \pm 0.07$ & $5.72 \pm 0.16$ & 19.16 & Dec. 99 \\
HIP74234 & 10.272 & 0.001 & 9.422 & 0.001 & 8.446 & 0.001 & $-1.28 \pm 0.07$ & $7.08 \pm 0.11$ & 33.68 & Dec. 99 \\
HIP74235 & 9.821 & 0.001 & 9.059 & 0.001 & - & - & $-1.30 \pm 0.07$ & $6.74 \pm 0.08$ & 34.14 & Dec. 99 \\
HIP81170 & 10.344 & 0.001 & 9.611 & 0.001 & 8.723 & 0.001 & $-1.14 \pm 0.07$ & $6.18 \pm 0.15$ & 20.71 & Dec. 99 \\
HIP3026 & 9.722 & 0.001 & 9.269 & 0.001 & 8.710 & 0.001 & -1.50 & 4.15 & 9.57 & Dec. 00 \\
HIP24316 & 9.924 & 0.001 & 9.448 & 0.001 & 8.838 & 0.001 & $-1.44 \pm 0.07$ & $5.28 \pm 0.15$ & 14.55 & Dec. 00 \\
\hline
\end{tabular}


Fig. 3. Residual between standard magnitudes and calibrated magnitudes for the Landolt stars in the Feb. 2002 run.

would have been needed to convert the values to the metallicity of M 5, and be distributed over a wide range of magnitudes and colors so as to overlap a sufficiently large portion of MS and ensure, in this way, a more robust fit to the cluster MS mean ridge line. The sample consists of 6 stars, 4 of which were observed in the December 1999 run, the others in December 2000. Each star has at least three measurements, to reduce the error on the magnitudes and remove the effect of eventual bad pixels and/or flat field inhomogeneities.

Since these stars needed to be observed with very short exposure times, the shutter delay of SUSI2 was taken into account. In particular, the typical exposure time was $0.5 \mathrm{~s}$, and the shutter delay for SUSI2 was taken from the SUSI2 web page at $\mathrm{ESO}^{2}$.

Table 2 shows the calibrated magnitudes and colors for the selected sample. The error indicated is the internal photometric error, and is not representative of the total error. To obtain the total error, uncertainties on the reddening and trigonometric

\footnotetext{
${ }^{2}$ http://www.ls.eso.org/Telescopes/NEWNTT/refdata/ param_ccd.html shows that the exposure is uniform down to $0.3 \mathrm{~s}$. Since the stars were placed close to the center of the chip, the resulting shutter effect turns out to be negligible.
}

parallax must be applied. As also pointed out by Gratton et al. (2003), these stars have virtually zero reddening. The error on the trigonometric parallax, given in the literature, was applied following the propagation of errors. The Lutz-Kelker correction was computed on the basis of the discussion by Carretta et al. (2000), by using the formula adopted by the authors (Eq. (1) in the cited work), because the sample had the same selection effect as the one reported in that paper. This value is of the order of $0.02 \mathrm{mag}$. The associated Malmquist bias, for the same reason, is negligible. By adding all the error sources, the average uncertainty increases to $\sim 0.03$ mag but with a spread given by star-to-star differences.

Since the selection was done with the criterium on the metallicity explained above and requiring that the objects be, of course, visible from the site and in the period of observation, only six subdwarfs have been selected, one of which saturated the $I$ filter even with the shortest possible exposure. The effect of metallicity on the subdwarf fitting to the distance has been extensively discussed by several authors in recent papers (see, e.g., Gratton et al. 1997; Carretta et al. 2000; Gratton et al. 2003; Reid 1997; Pont et al. 1998) and will not be readdressed here. However, by selecting objects with a metallicity close to the cluster's, the uncertainities due to the metallicity spread are reduced, and the correction to a common metallicity value is small. The metallicity of the cluster has been recently recalibrated in the CG97 scale as $[\mathrm{Fe} / \mathrm{H}]=-1.10$, while ZW84, and subsequent updates, give an estimate of $[\mathrm{Fe} / \mathrm{H}]=-1.40$. The metallicity scale of the subdwarfs, instead, has been recalibrated by Gratton et al. (2003) and found to be, on average, $0.13 \pm 0.04$ dex smaller than the one given in Carretta et al. (2000). This offset is to be taken into account in the DM determination, and will be discussed below.

\section{The color-magnitude diagram}

\subsection{The NTT CMD}

The CMD derived from ground-based data (February 2002) is shown in Fig. 4. The diagram extends from the upper part of the RGB down to about $V \sim 24$. A reasonably large magnitude range on the MS is crucial to convert the HST magnitudes (both VEGAMAG and STMAG) to the ground NTT system. The CMD from the December 1999 run was obtained under relatively poor seeing conditions and hence was not suitable to perform an accurate calibration of the HST data to the ground system. For this reason we adopted the February 2002 data-set 


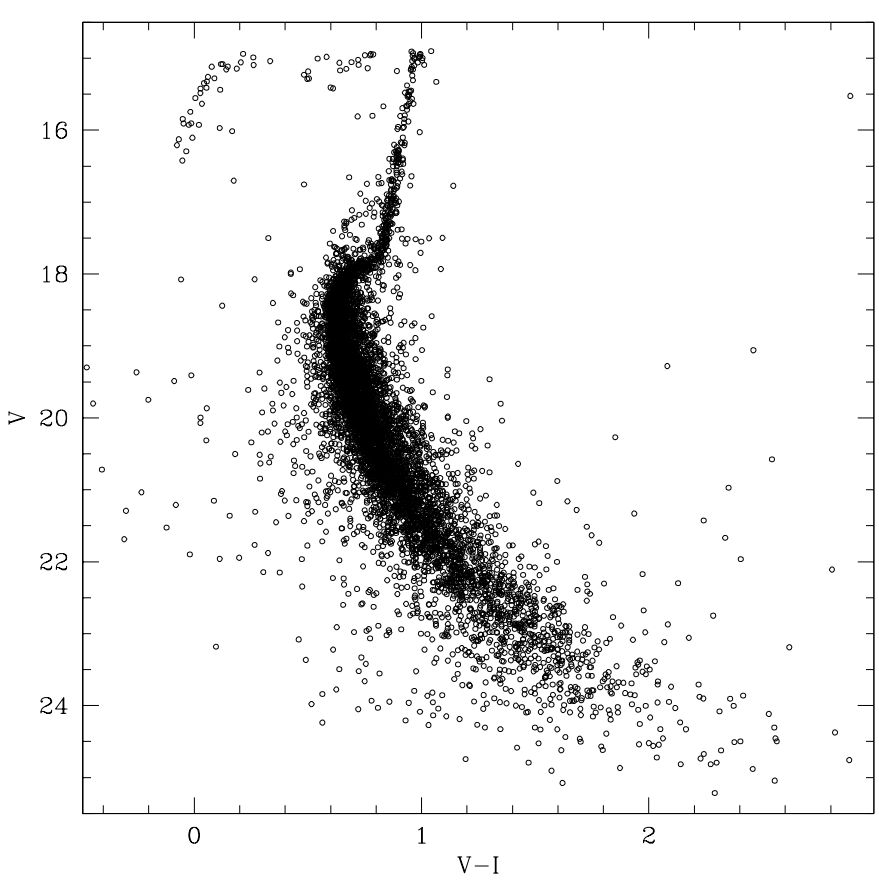

Fig. 4. Ground CMD.
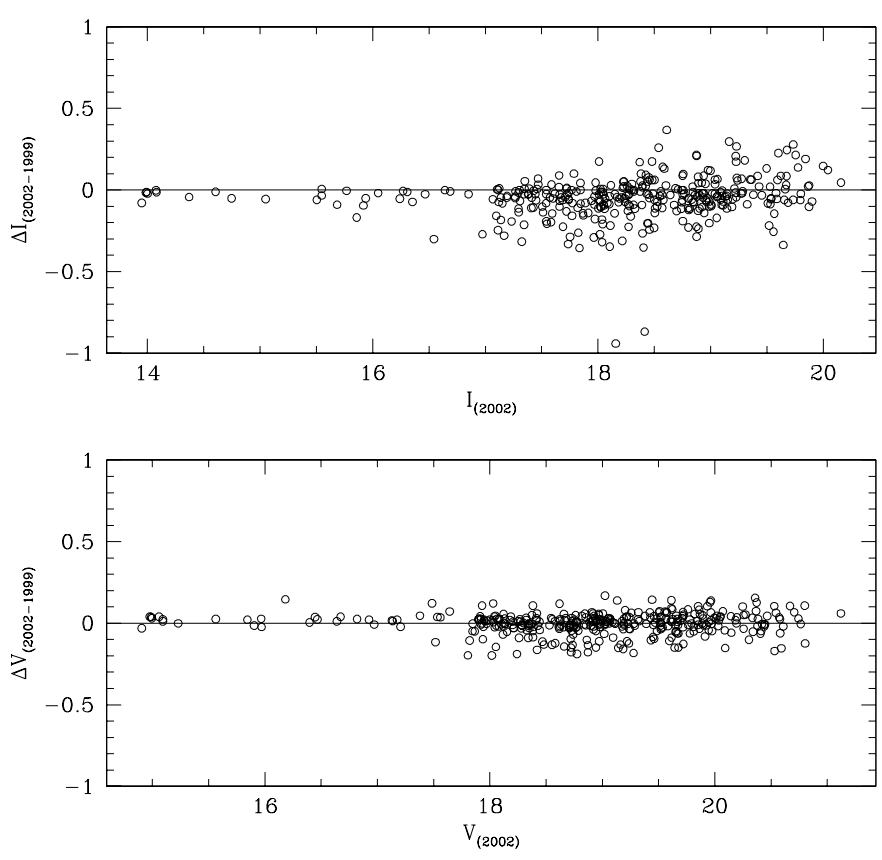

Fig. 5. Run to run scatter in the ground measurements.

after checking that the two sets of measurements were selfconsistent. Figure 5 shows the results of the check.

\subsection{The HST CMD}

The HST measurements were averaged, recording also information on the number of frames in which each object was detected in each filter. As a final catalog we consider all the stars appearing in at least $80 \%$ of the frames (at least $5 \mathrm{~V}$ and $7 \mathrm{I}$ ). This choice is a good compromise between taking all the measurements and taking only the stars detected in all the frames.



Fig. 6. CMD from HST data.

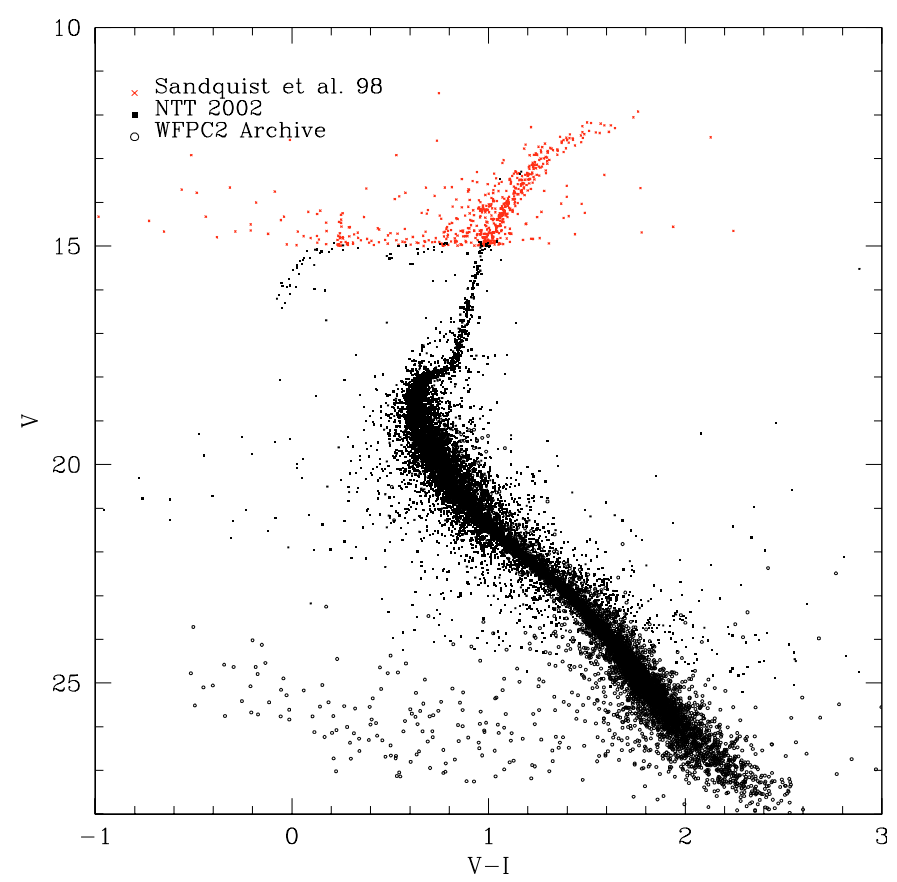

Fig. 7. Final CMD.

The first choice would contaminate the CMD with all the spurious detections that appear by chance on only one frame. The second leads to missing faint objects, like WDs. The brighter part of the catalog (down to $V \sim 26.5$ ) is not altered by this selection criterion, as expected. The HST CMD is reported in Fig. 6, where we show only the VEGAMAG magnitudes.

The whole M 5 CMD is reported in Fig. 7. The HST dataset extends approximately from $V=19.5$ to $V=28$, but the top of the sequence is spread out because the chip is close to saturation and will not be considered in the following analysis. $F 555 \mathrm{~W}$ and $F 814 \mathrm{~W}$ magnitudes were converted to the 
ground-based CMD via a relative calibration equation of the form (for the $V$ magnitude, but it applies to the $I$ magnitudes as well)

$V_{\text {ground }}=V_{\mathrm{HST}}+z p+c t \times\left(V_{\text {ground }}-I_{\text {ground }}\right)$.

The output for the $V$ magnitude turned out to be identical for STMAG as well as for VEGAMAG, while for the $I$ filter we derived, as expected, different transformations. The two HST magnitude systems have been checked for selfconsistency after transforming them to the ground NTT system. The two transformed magnitudes still show some residuals that, over a range $20<V<23$ is $\sim 0.01 \mathrm{mag}$, with a shape that resembles that of a CMD. This is an indication that the color effects were not completely eliminated by the transformations, probably because of the larger scatter in the groundbased CMD and the relatively small magnitude overlap of the ground- and space-based data. However, even at fainter magnitudes, the overall difference between the two magnitudes is always below $0.05 \mathrm{mag}$ (at $V \sim 27.5$ ). In the following, we will adopt as HST-transformed magnitudes the ones coming from VEGAMAG, because this system is closer to the ground Vega system, so that the required transformation is smaller and thus less prone to residual uncertainties ${ }^{3}$.

\subsection{Mean ridge line}

The mean ridge line has been drawn on the faint part of the CMD by using HST data, on the upper MS until the sub-giant branch with the NTT plus literature data, on the upper part of the RGB and the HB with the (Rosenberg et al. 2000, R00) data, since their RGB is very narrow and well populated. In general, to build the ridge line the CMD was "sliced" in magnitude bins and, for each bin, the median point of the distribution was determined, together with the associated scatter. Only the objects with the lowest errors have been selected, to ensure that the uncertainty on the ridge is small. The zones with a strong curvature, i.e. at the bottom of the SGB, were treated differently, by applying a polynomial fit to that range and projecting the stars along the curved abscissa, then re-fitting iteratively the polynomial. In this way, the CMD is sliced along the curve and it is possible to find the median point with high accuracy also in the most bent magnitude bins. Figure 9 shows the ridge line of the lower MS with its uncertainty strip. The narrowness of the uncertainty strip will be used later to constraint the fit to the subdwarf sequence. As a comparison term, Fig. 8 shows the ridge line determined as described above, the fiducial by S96 and the ones derived with the same method by using the data of Johnston \& Bolte (1998) and R00, that are in close agreement with ours. The irregularities in the ridge lines obtained with literature data, that can be seen at the base of the RGB, are due to the small size of the magnitude bins chosen to draw the fiducial. Since we are not interested in a careful analysis of those ridge lines, but only show them for sake of comparison, we did not apply any smoothing or more refined technique to improve it. However, our fiducial line extends about $5 \mathrm{mag}$

\footnotetext{
${ }^{3}$ See also the "HST Data Handbook for WFPC2" at http:// www.stsci.edu/hst/HST_overview/documents/datahandbook
}



Fig. 8. Comparison of ridge lines for M 5 .

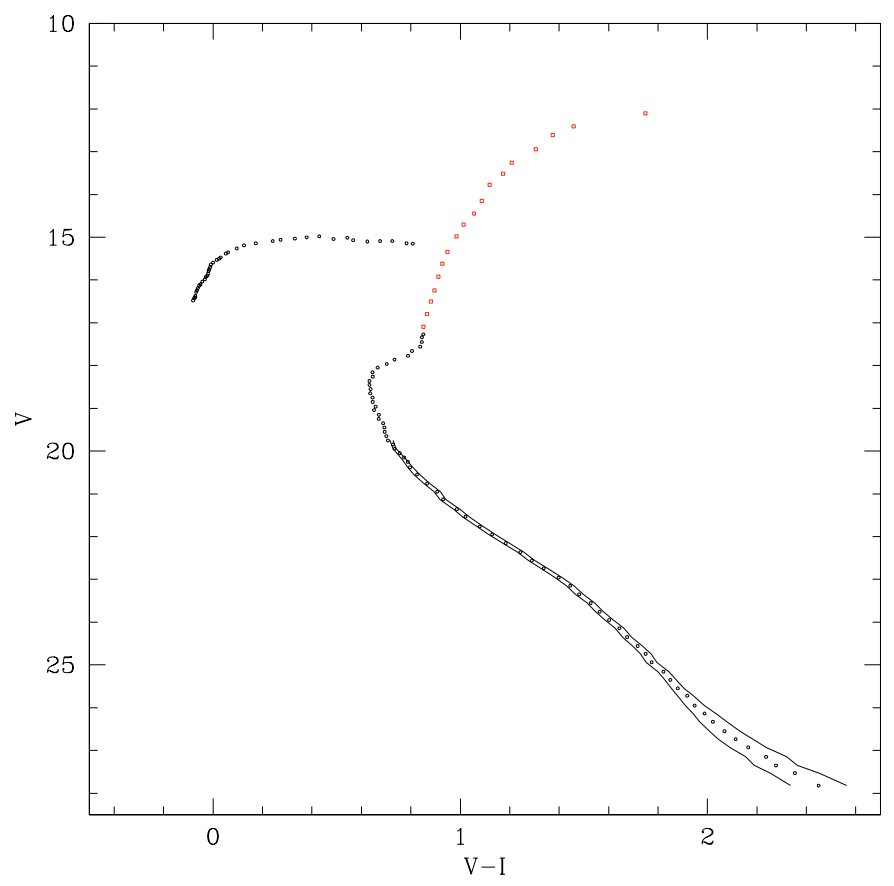

Fig. 9. The ridge line of the lower MS of M 5 with its uncertainty strip.

below previous works. This fact is crucial because, at least in principle, it allows us to check model predictions down to the bottom of the MS, as well as to use very faint subdwarfs to constrain the distance fitting, if any (see below).

\subsection{Comparison with previous photometry}

Beside the comparison of the ridge lines, a further check has been done by comparing the NTT CMD with recent literature results, namely R00, Johnston \& Bolte (1998) and S96. 


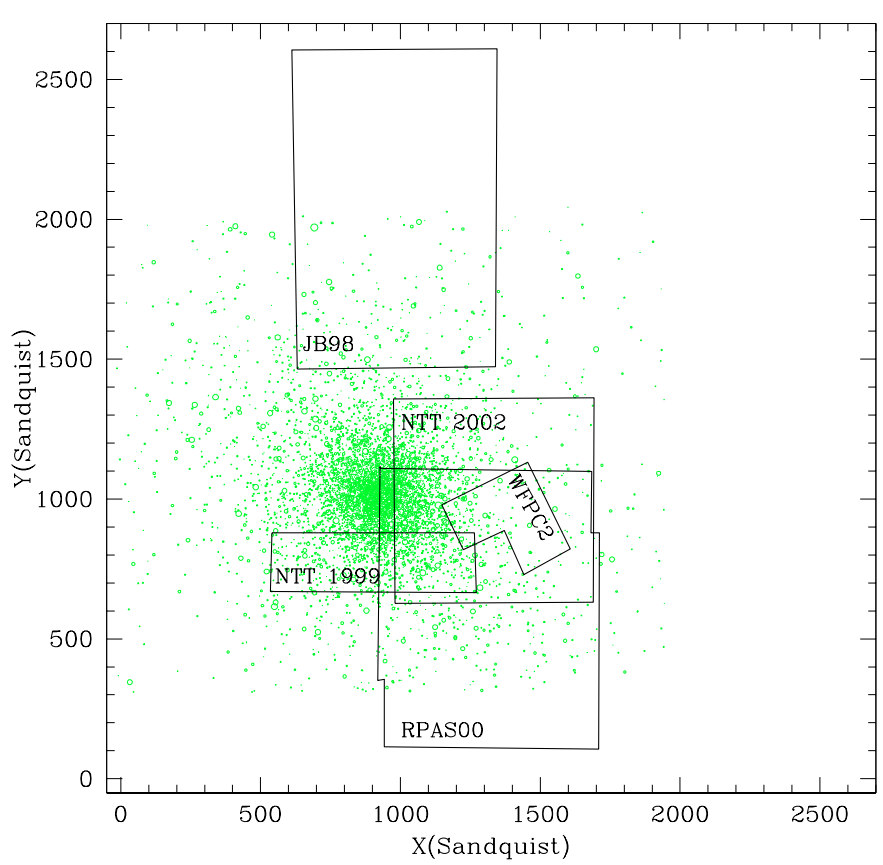

Fig. 10. Map of the fields covered by this work and recent literature.
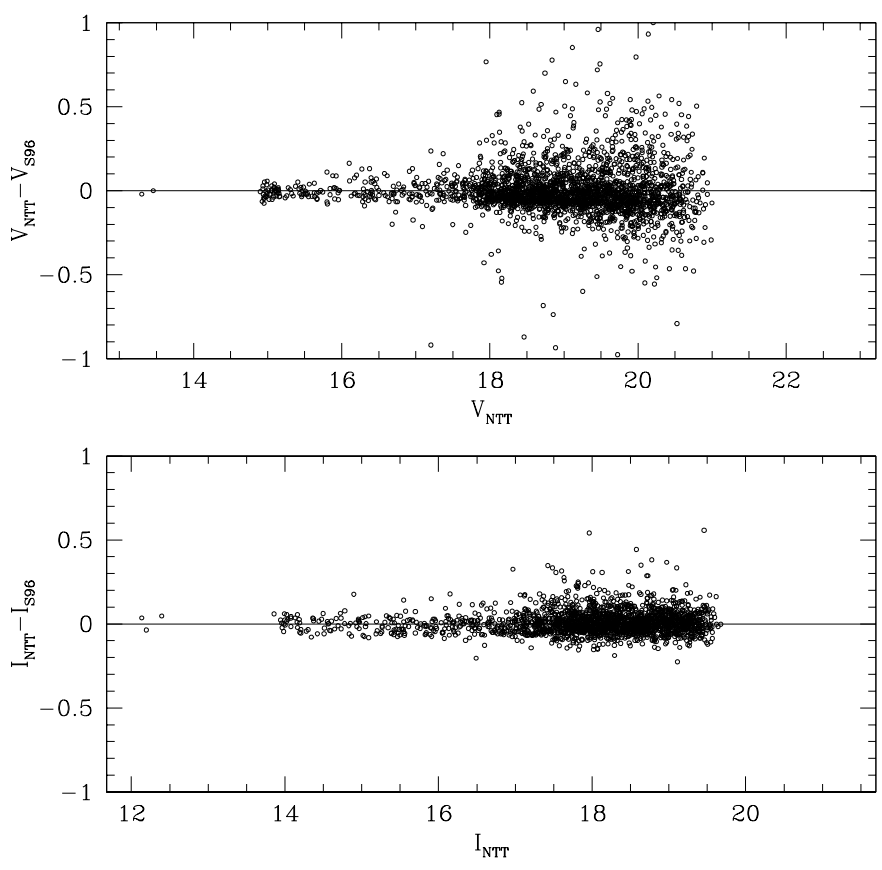

Fig. 11. Comparison with the data-set of S96.

Figure 10 shows the positioning of fields observed by us, the HST pointing, and the three comparison works. The plots in Figs. 11 and 12 show the comparison between our magnitudes and the cited ones, where an overlapping of the fields permitted a direct match of the objects. Our data show a good agreement with S96 and R00 but the average scatter is quite large, meaning that a trend with position could be present. Actually, there is a small residual trend with position, with an amplitude of the order of $0.02 \mathrm{mag}$. In the comparison with the two literature photometries cited above the trend goes in opposite directions, in the sense that the trend along the $X$-coordinate, in the reference system of $\mathrm{S} 96$, resembles the trend along the $Y$-coordinate
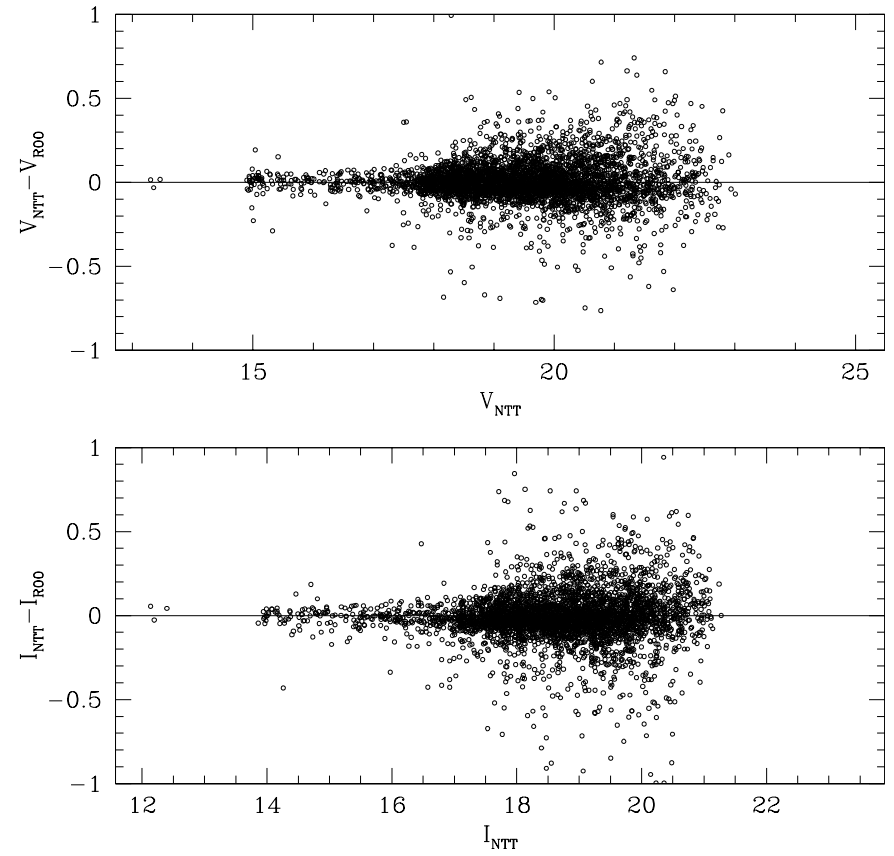

Fig. 12. Comparison with the data-set of R00.

in the R00 data-set, always in the reference system of S96 (all the data have been reported to the system of S96), and vice-versa. The amplitude of the trend is larger with S96 than with R00, and is absent if we compare the NTT 1999 data with both S96 and R00. The only valid explanation for this is that a residual flat-field is still present in some data-sets with respect to the others. In fact, in the reduction of the February 2002 data-set, we applied a more refined technique for flat-fielding the data, described in Hainaut (1998), which takes into account also the lowest spatial frenquencies. The 1999 data-set has not been corrected for this effect. On average, this removed a residual trend in the fluxes. Since this procedure has been applied with success in other works (Monelli et al. 2004, in preparation), we find no reason to discard the procedure or re-calibrate our data in the system of S96 or R00. However, the effect is visible especially at fainter magnitudes $(V>19)$, while is almost absent on the RGB.

\section{Distance via subdwarfs fitting}

\subsection{Error sources}

Before proceeding with the DM fitting, it is appropriate to discuss the contribution of the various error sources to the total uncertainty on the DM. Gratton et al. (2003) report in their Table 1 a detailed list of all the error budget contributors before and after the re-analysis done in that paper. Taking that as a guideline, we can now see how the various uncertainties combine in our case. In Sect. 4 we mentioned some of the typical uncertainties in the subdwarf analysis, and the fact that, since our sample has been drawn from the same lists published by Gratton et al. (1997); Carretta et al. (2000); Reid (1997) and, partially, Gratton et al. (2003), we expect that the uncertainty given by the Lutz-Kelker effect, the Malmquist bias and, clearly, the parallax, are essentially the same. Hence we adopt the values given in Table 1 of Gratton et al. (2003). 


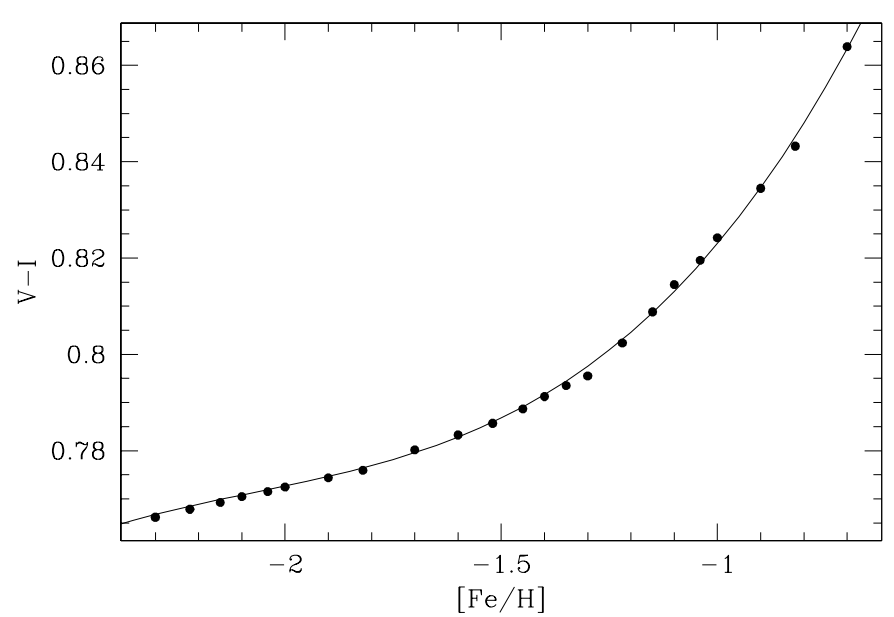

Fig. 13. Color-metallicity relation for the $V-I$ color, taken from the models of Straniero et al. (1997).

Photometric errors are very small both for the subdwarfs (see Table 2) and the cluster ridge line. For this last, in particular, the very precise HST photometry coupled with a good ground-based calibration allowed us to obtain an average error on the ridge line of the order of $0.005 \mathrm{mag}$ at $(V-I)=0.7$. The error from the calibration relation is also very small and of the order of $0.02 \mathrm{mag}$, since the photometric conditions of the calibration night were close to excellent. Subdwarfs and cluster have been observed in the most homogeneous possible conditions and thus, the errors on the photometric calibration are expected to be very small. A residual systematic trend could be present if the calibrated magnitudes were not reported to the standard system, but the obtained values are compliant with all the existing results, and thus we discard this possibility.

Three other sources of error have to be now examined: the metallicity scale, the cluster's reddening, the presence of binaries. Their contribution affects both random and systematic errors.

\subsubsection{Metallicity}

The metallicity scale is a major issue and has been widely investigated in previous works (see e.g., Gratton et al. 2003; Carretta et al. 2000; Reid 1997; Pont et al. 1998). There are two main aspects to take into account: i) the spread in metallicity given by the distribution in $[\mathrm{Fe} / \mathrm{H}]$ of field stars; ii) the relative metallicity scale used. In order to reduce the first effect, the usual procedure is to apply a relation that transforms the values to a common metallicity (the cluster's). In our case we decided to use only stars having metallicity values as close

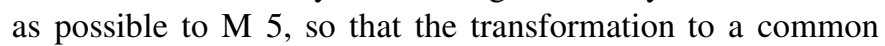
metallicity is small. In order to estimate it, we used the relation between $[\mathrm{Fe} / \mathrm{H}]$ and $(V-I)$ at $M_{V}=+6$, taken from the models of Straniero et al. (1997), that is shown in Fig. 13. A third order polynomial has been found to fit adequately the $(V-I)$ vs. $[\mathrm{Fe} / \mathrm{H}])$ relation in the range $-2.3<[\mathrm{Fe} / \mathrm{H}]<-0.5$. The second effect comes from the systematic difference between the two most commonly used scales, the ZW and the CG. Gratton et al. (1997) use a homogeneous metallicity system, but our subdwarf sample has been taken from that work and from Reid (1997), who used the ZW scale. Since our cluster has metallicity estimates $[\mathrm{Fe} / \mathrm{H}]=-1.1$ in the CG scale, and $[\mathrm{Fe} / \mathrm{H}]=-1.4$ in the $\mathrm{ZW}$ scale, we found it appropriate to transform Reid (1997) values to Gratton et al. (1997), i.e. from ZW to CG, and then selecting stars from the Reid (1997) sample with the reported metallicity close to the cluster's one.

It should be noted that some of the stars in the present sample have not been recalibrated by Gratton et al. (2003), hence a residual systematic spread might be present which affects the DM fitting as well, due to the co-existence of two slightly different recalibrations. If the value of $0.13 \mathrm{dex}$ is taken as an average recalibration offset, we estimate a residual systematic error due to metallicity of \pm 0.008 (at $1 \sigma)$ in the $(V-I)$. Given the slope of the MS between $M_{V}=+5$ and $M_{V}=+6$, this propagates into an uncertainty of $0.04 \mathrm{mag}$. The uncertainity on the metallicity (usually $0.1 \mathrm{dex}$ ) is, instead, added to the random error budget and corresponds, following Gratton et al. (2003), to \pm 0.08 mag.

\subsubsection{Reddening}

The cluster's reddening also plays a double rôle, since the associated error affects the global random error and the uncertainty on the absolute amount of reddening is a source of systematic error. We shall discuss this point quantitatively below when we present the fitting procedure.

\subsubsection{Binaries}

The presence of binary stars affects both the subdwarf and the cluster sample. Although we selected bona fide single stars, a residual quantity of binaries could still affect the subdwarf sample. We rely always on the fact that the sample has been selected from the published lists of Gratton et al. (1997) and Reid (1997), and thus assume their figure for the contribution of subdwarf binaries to the total error, i.e. \pm 0.02 (see Gratton et al. 2003). The effect of binaries on the cluster ridge line is slightly more complicated. The effect of the presence of binaries in the cluster is to spread the main sequence toward redder colors and, in extreme cases, to draw a separate sequence, as has been detected in some open clusters (see, e.g., Marconi et al. 1997). In our case, the HST main sequence is very narrow, and a welldefined clear binary sequence would be visible. Since this is not the case, we tried to estimate the effect of an MS spread due to an unresolved sequence of binaries. To do this, we "rectified" the main sequence and built a color histogram of the brightest portion of the HST MS, that has a uniform width over the considered range $(20<V<22)$. A Gaussian profile has been fit to this profile, considering only the blue tail of the distribution, that is in principle unaffected by the presence of binaries. The fitted profile has then been subtracted from the original distribution. The residual red tail is an indication of the binary contribution. This procedure is quite robust and gives reliable figures for the uncertainties on the position of the MS locus in the CMD. From this, we derive an estimate of a possible systematic deviation of the MS ridge line from the "true" single 




Fig. 14. Fit of the cluster fiducial to the subdwarf sequence. The stars with the label on the right belong to the observed sample. The others have been taken from published works.

star MS given by binaries of $\sigma(V-I)=0.005$, in addition to the random error due to the calculated spread of the MS. Again, this value in color propagates into a \pm 0.025 mag of systematic uncertainty. Table 4 summarizes all the error sources involved in the DM fitting process.

\subsection{Distance modulus fit}

Taking into account all the considerations and the caveats described above, we used the subdwarfs sample to fit a DM to the cluster data. In order to do this, we adopt a value of reddening of $E(B-V)=0.035( \pm 0.005)$ (Carretta et al. 2000 , and references therein), corresponding to $E(V-I)=$ 0.056, (obtained from the relation in Rieke \& Lebofsky (1985): $E(V-I)=1.6 \times E(B-V))$. The quoted uncertainty has been taken from the literature, and propagates into a 0.025 uncertainty in magnitude. The DIRBE maps (Schlegel et al. 1998) report a reddening value in the direction of $M 5$ of $E(B-V)=0.036$, consistent with the absolute value given in Carretta et al. (2000). As shown in Fig. 14, the brightest calibrating subdwarfs (HIP3026 and HIP24316) turn out to be too blue to fit directly the cluster ridge line, because the cluster MS has already evolved toward the turn-off point. However, they are useful to draw the subdwarf main sequence locus to be compared with our data. Of the other three stars, HIP81170 is known to be a spectroscopic binary, and this might justify its redder color. The other two, HIP70681 and HIP74234, have the smallest error and were used to fit the cluster ridge line. We fit the cluster fiducial line to the subdwarf sequence by shifting the fiducial according to the reddening (in $(V-I)$ !) and extiction $-3.1 \times E(B-V)-$, then with a least square fitting of the difference between the subdwarfs used and the cluster
Table 3. Extra subdwarfs from the literature.

\begin{tabular}{lrrrr}
\hline \hline Ident. & $M_{V}$ & $(V-I)^{a}$ & {$[\mathrm{Fe} / \mathrm{H}]$} & Source \\
\hline HIP57939 & $6.61 \pm 0.02$ & 0.88 & $-1.33 \pm 0.07$ & G03, H \\
HIP57450 & $5.59 \pm 0.25$ & 0.63 & $-1.26 \pm 0.07$ & G97, H \\
HIP74235 & $6.74 \pm 0.08$ & 0.81 & $-1.38 \pm 0.07$ & G03, H \\
HIP79537 & $6.84 \pm 0.02$ & 0.94 & $-1.39 \pm 0.13$ & G97, H \\
HIP7459 & $5.43 \pm 0.23$ & 0.679 & -1.2 & R01 \\
HIP73798 & $5.90 \pm 0.26$ & 0.727 & -1.2 & R01 \\
HIP89215 & $6.50 \pm 0.26$ & 0.86 & -1.2 & R01 \\
HIP100568 & $5.44 \pm 0.12$ & 0.678 & $-1.00 \pm 0.07$ & R01, G03 \\
\hline
\end{tabular}

${ }^{a}$ G97: Gratton et al. (1997); H: HIPPARCOS catalog, for $V-I$ color; R01: Reid et al. (2001); G03: Gratton et al. (2003).

Table 4. Contribution to the total uncertainty, in magnitudes, of the various error sources. Values are at the $1 \sigma$ level.

\begin{tabular}{lcc}
\hline \hline & Field stars & Cluster \\
\hline Photometry & \pm 0.001 & \pm 0.005 \\
Calibration & \pm 0.02 & \pm 0.02 \\
Lutz-Kelker & \pm 0.02 & - \\
Malmquist & Negligible & - \\
Parallax & \pm 0.01 & - \\
Metallicity (rand.) & \pm 0.08 & \pm 0.08 \\
Metallicity (syst.) & \pm 0.04 & \pm 0.04 \\
Reddening (rand.) & Negligible & \pm 0.025 \\
Reddening (syst.) & Negligible & \pm 0.03 \\
Binaries & \pm 0.02 & \pm 0.025 \\
\hline
\end{tabular}

fiducial, with their errors. Figure 14 shows the results of the best fit, $\mu_{0}=14.44$. If a value of $E(B-V)=0.03$ is adopted, which corresponds to $E(V-I)=0.048, \mu_{0}=14.41$ is obtained. The error on the fit obtained from the combination of the random error sources only is, in both cases, \pm 0.09 , the uncertainty in the metallicity being the strongest contributor. To increase the number of subdwarfs suitable to form a fiducial reference sequence, we added to our sample other subdwarfs listed in Gratton et al. (1997) and Reid et al. (2001), having $[\mathrm{Fe} / \mathrm{H}]$ similar to that of $\mathrm{M} 5$ and discarding known double stars. The added stars are listed in Table 3 and are shown with different symbols (filled circles) in Fig. 14. It can be seen that most of the added stars draw a good reference main sequence, with the exception of HIP57450 and HIP74235, that are considerably bluer. On the $(V, B-V) \mathrm{CMD}$, however, they lie on the MS, and thus we are inclined to interpret their bluer colors as uncertainties in the $I$ magnitudes, that have been taken from the original HIPPARCOS catalog. For this reason, the two outlying stars have been excluded from the fit.

The added stars define three well separated sequences corresponding to the three metallicity ranges selected, from the lowest (triangles, bluest), to the intermediate (circles), to the highest (squares, reddest).

With the extended sample of subdwarfs, our best estimate for the (dereddened) DM is $\mu_{0}=14.44$ if $E(B-V)=0.035$ is adopted. If, instead, $E(B-V)=0.03$ is adopted, as reported in 
Gratton et al. (1997), the DM turns out to be $\mu_{0}=14.41$, thus confirming the value obtained with the original, and homogeneous, sample.

The error associated with the fit above has been estimated from all the error sources discussed above and reported in Table 4, considering random and systematic errors separately. From a statistical error propagation over the involved quantites, the uncertainty associated with the fit is $\pm 0.09 \mathrm{mag}$. A systematic uncertainty of $\pm 0.07 \mathrm{mag}$, combined from the reddening and metallicity scale, must then be added. The global uncertainty is slightly larger than the one reported by Gratton et al. (2003), probably because the $V-I$ color is more sensitive to metallicity variation and thus the effects of variation in $[\mathrm{Fe} / \mathrm{H}]$ and $E(B-V)$ are amplified with respect to the $B-V$ color.

\section{Discussion and conclusions}

This study aimed to estimate the DM of M 5 through the mainsequence fitting method, using as the calibrating sequence a set of local subdwarfs for which the trigonometric parallax from HIPPARCOS was known. To achieve our goal we used the same setup of telescope+instrument+filters both for the cluster and subdwarf data, namely the ESO-NTT equipped with the camera SUSI 2 and standard ESO $V$ and $I$ filters.

Later, a set of subdwarfs from the literature was added to the original sample to improve the sample size, and hence increase the accuracy of the fit. Comparisons with recent data from the literature show that our data are fully consistent with previous determinations, whose accuracy is known, both for the cluster and the local subdwarfs. A final sample of $\sim 10$ subdwarfs was used to fit a DM to the cluster. By taking into account the two most commonly accepted values for the reddening (i.e. $E(B-V)=0.03$ and 0.035$)$, we obtained two very close values for the (dereddened) DM: $\mu_{0}=14.41 \pm 0.09 \pm 0.07$ and $\mu_{0}=14.44 \pm 0.09 \pm 0.07$, respectively, where the first number comes from random error sources, the second from systematic error sources. These values are in good agreement both with Reid (1997) $\left(\mu_{0}=14.45\right.$ obtained with the subdwarfs having the smallest error on the parallax) and Carretta et al. (2000) ( $\mu_{V}=14.59$ corresponding to $\mu_{0}=14.48$ ), with a slight preference for the shorter value. Vandenberg et al. (2002), instead, found $\mu_{0}=14.38$ with the use of non-diffusive isochrones and $\mu_{0}=14.30$ with diffusive isochrones. In their work, they use a reddening value $E(B-V)=0.038$ and a metallicity $[\mathrm{Fe} / \mathrm{H}]=-1.4$ for the cluster, discussing their preference for the $\mathrm{ZW}$ scale over the $\mathrm{CG}$, arguing that the metallicity for M 5 is inconsistent with the metal determination for the field stars, if the value is as high as -1.1 . Moreover, Vandenberg et al. (2002) chose to not normalize the field stars to a common metallicity, but this effect acts on the spread of the relation rather than on the absolute value.

To obtain a further constraint on the DM, we tried to use a set of extreme subdwarfs, using an analogous approach to Reid \& Gizis (1998), that has been found to be inapplicable because both the main sequence locus at the faint magnitude level of the extreme subdwarfs $\left(M_{V} \sim 10\right)$ is dispersed and the subdwarfs of intermediate metallicity define a sequence dispersed as well. To reduce the uncertainty, it would have been desirable to have a set of subdwarfs of fainter magnitudes (i.e. below $M_{V} \sim 7$ ) to take full advantage of the narrowness of the HST main sequence, but they are lacking in the HIPPARCOS sample. Moreover, at such faint magnitude levels, the lower main sequence stars are very cool and the determination of their metallicity much more uncertain because it is very difficult to take into account all the molecular species in the atmosphere at these low temperatures.

Acknowledgements. We wish to thank Drs. Eric Sandquist and Jennifer A. Johnston for giving us their published tables in electronic form and Dr. Olivier R. Hainaut for useful and pleasant discussions. We would also like to thank the referee, Dr. R. Gratton, for his useful comments that improved the presentation of this paper. This work has been partially supported by the Italian Ministry of Education, University and Research (MIUR) under grant "COFIN 2000".

\section{References}

Carretta, E., \& Gratton, R. G. 1997, A\&AS, 121, 95

Carretta, E., Gratton, R. G., Clementini, G., \& Fusi Pecci, F. 2000, ApJ, 533, 215

Chaboyer, B., Demarque, P., Kernan, P. J., \& Krauss, L. M. 1998, ApJ, 494, 96

Dolphin, A. E. 2000, PASP, 112, 1397

Gratton, R. G., Fusi Pecci, F., Carretta, E., et al. 1997, ApJ, 491, 749

Gratton, R. G., Bragaglia, A., Carretta, E., et al. 2003, A\&A, 408, 529

Hainaut, O. R., Meech, K. J., Boehnhardt, H., \& West, R. M. 1998, A\&A, 333, 746

Holtzman, J. A., Hester, J. J., Casertano, S., et al. 1995, PASP, 107, 156

Johnston, J. A., \& Bolte, M. 1998, AJ, 115, 693

Lutz, T. E., \& Kelker, D. H. 1973, PASP, 85, 573

Marconi, G., Hamilton, D., Tosi, M., \& Bragaglia, A. 1997, MNRAS, 291, 763

Perryman, M. A. C., Lindegren, L., Kowalewsky, J., et al. 1997, A\&A, 323, 49

Pont, F., Mayor, M., Turon, C., \& Vandenberg, D. A. 1998, A\&A, 329, 87

Ramirez, S. V., \& Cohen, J. G. 2003, AJ, 125, 224

Reid, I. N. 1997, AJ, 114, 161

Reid, I. N., \& Gizis, J. E. 1998, AJ, 116, 2929

Reid, I. N., van Wyk, F., Marang, F., et al. 2001, MNRAS, 325, 931

Renzini, A. 1991, in Globular Cluster Ages and Cosmology, ed. T. Shanks, A. J. Banday, \& R. S. Ellis, Observational Tests of Cosmological Inflation, NATO-ASI Ser. C, 348, 131

Rieke, G. H., \& Lebofsky, M. J. 1985, ApJ, 288, 618

Rosenberg, A., Piotto, G., Aparicio, A., \& Saviane, I. 2000, A\&AS, 145, 451 (R00)

Sandquist, E. L., Bolte, M., Stetson, P. B., \& Hesser, J. E. 1996, ApJ, 470, 910 (S96)

Schlegel, D. J., Finkbeiner, D. P., \& Davis, M. 1998, ApJ, 500, 525

Straniero, O., Chieffi, A., \& Limongi, M. 1997, ApJ, 490, 425

Tody, D. 1986, The IRAF Data Reduction and Analysis System, in Proc. SPIE Instrumentation in Astronomy VI, ed. D. L. Crawford, 627, 733

Tody, D. 1993, IRAF in the Nineties, in Astronomical Data Analysis Software and Systems II, ASP Conf. Ser., 52, ed. R. J. Hanisch, R. J. V. Brissenden, \& J. Barnes, 173

Vandenberg, D. A., Richard, O., Michaud, G., \& Richer, J. 2002, ApJ, 571,487

Zinn, R., \& West, R. M. 1984, ApJS, 55, 45 